\title{
ATP bioluminescence values are significantly different depending upon material surface properties of the sampling location in hospitals
}

Tomoko Shimoda', Rika Yano ${ }^{1}$, Shinji Nakamura², Mitsutaka Yoshida², Junji Matsuo ${ }^{3}$, Sadako Yoshimura ${ }^{1}$ and Hiroyuki Yamaguchi ${ }^{3 *}$

\begin{abstract}
Background: Our previous study into assessing hospital cleanliness in Japan by two common methods, ATP bioluminescence and the stamp agar method, revealed considerable variability in the data of both methods (BMC Research Notes, $7: 121,2014)$. To investigate the reason(s) for the variability, we reanalyzed the data $(n=752)$ from the point of view of the material surface properties of sampling sites.

Methods: Data obtained from surfaces with unknown properties and different purposes such as floor were omitted, and the remaining data $(n=488)$ were used for this study. The material surface properties on sampling sites were divided into six categories: melamine coated $(n=216)$, vinyl chloride $(n=16)$, stainless steel $(n=144)$, wood $(n=63)$, and acrylonitrile-butadiene styrene resin coated $(n=48)$. The data between individual material properties were compared.

Results: The ATP values of high-touch places were significantly different depending on the type of surface, but no significant difference in stamp values between material properties was seen, indicating that in contrast to stamp values, ATP-accumulation more depends on the physical properties of the material surface such as electronic charges or roughness. To confirm this, we assessed a degree of roughness on vinyl chloride material surface (disutilized floor samples actually used for each of the hospitals) by observation with scanning electron microscope (SEM). As a result, SEM observation similarly revealed considerable roughness on the materials, which may allow microbes to contaminate the materials without noticing it.
\end{abstract}

Conclusion: Material properties must be considered when evaluating hospital cleanliness with ATP values, and provide a strong warning into evaluating hospital cleanliness.

Keywords: Material properties, Hospital cleanliness, ATP bioluminescence, Stamp agar, Culture, Scanning electron microscopy

\section{Background}

In the last decade, much attention has been focused on hospital-acquired infections caused by microbecontaminated hospital environments. Surfaces that are

\footnotetext{
*Correspondence: hiroyuki@med.hokudai.ac.jp

${ }^{3}$ Department of Medical Laboratory Science, Faculty of Health Sciences, Hokkaido University, Nishi-5 Kita-12 Jo, Kita-ku, Sapporo, Hokkaido 060-0812, Japan

Full list of author information is available at the end of the article
}

frequently touched by hands, such as doorknobs, sinks, lockers, and tables of inpatients, presumably provide the greatest risk for patients [1-3]. Consequently, improving hand hygiene and isolation practices are critically important to control hospital-acquired infections, and routine cleaning practices in hospitals has been responsible for a decrease in transmission of hospital-acquired infections [4-7]. Hospital cleanliness protocols, according to guidelines such as the US. Centers for Disease 
Control and Prevention, include appropriate monitoring of hospital environments with a warning that visual assessment of hospital cleanliness is not enough to control hospital-acquired infections [8-10]. For monitoring hospital cleanliness, both the adenosine triphosphate (ATP) bioluminescence (ATP method), which is an indicator of general organic contamination [11-15], and the standard stamp agar method (stamp method), which is an indicator of microbiological contamination [16-18], has been used worldwide.

We have previously assessed hospital cleanliness using the ATP and stamp methods: the surfaces of 752 sites in nurse and patient areas in three hospitals located in a central area of Sapporo, Japan were evaluated by both methods (each of the surfaces was sampled eight times in 2 months). The results revealed the presence of a wide range of organic contamination spread via hand touching, including microbial contamination [19]. However, both methods indicated considerable variability regardless of daily visual assessment and wiping, and positive surfaces were irregularly seen, suggesting that ongoing daily hospital cleanliness is insufficient, although the exact reason for the variability remains unclear [19].

Several previous studies have suggested that in dental or food sciences, material surface properties influence microbial contamination [20-22]. In the present study, we reanalyzed the data from our previous study $(n=752)$ from the point of view of the material surface properties of the sampling sites. We show that material surface properties must be considered when evaluating high-touch places with ATP values, and provide a strong warning into overestimating hospital cleanliness.

\section{Methods}

\section{Data used for this study}

The 752 surfaces within nurse [instillation preparation table (nurse station); routine worktable (nurse station); nurse wagon (mobile station in nurse area); doorknob (nurse station)] and patient areas [guardrail in corridor (public space); hospital entrance floor (public space); locker for hospital inpatients (room with multiple beds); overbed table (room with multiple beds); locker for hospital inpatient (room with private bed); overbed table for hospital inpatient (room with private bed); windowsill (room with private bed); windowsill (room with multiple beds)] in three hospitals ["A" hospital ( $>500$ beds), "B" hospital (100-500 beds), and "C" hospital ( $<100$ beds)] located in a central area of Sapporo, Japan, have previously been evaluated by the ATP and stamp methods [19]. The dataset from this publication was re-used for the current study. As mentioned in the previous report, ATP bioluminescence was measured using a $3 \mathrm{M}$ Clean-Trace ATP System $\left(10 \times 10 \mathrm{~cm}^{2}\right.$; Sumitomo 3M Limited, Tokyo, Japan), and the values were expressed as bioluminescence relative light units (RLU) [19]. Wiping the area of each surface was performed with a cotton swab supplied with the system. Also, the commercial stamp agar assay was based on soybean casein digest $\left(10 \mathrm{~cm}^{2}\right.$ surface area; Nissui Pharmaceutical Co., Ltd., Tokyo, Japan) for monitoring environments for microorganism contamination; the agar plate was cultured for 5-7 days under aerobic conditions at $30{ }^{\circ} \mathrm{C}$, colonies were counted and these data were estimated as colony-forming unit (CFU) per $10 \mathrm{~cm}^{2}[19]$.

\section{Classification into individual material properties}

We contacted the manufacturers of various surfaces to determine the surface material properties. The data were divided into six categories: melamine coated $(n=216)$, vinyl chloride $(n=56)$, stainless steel $(n=144)$, wood $(n=63)$ and acrylonitrile-butadiene styrene resin coated $(n=48)$. Data obtained from unknown material properties $(n=200)$ and objects of different purpose (floor) $(n=64)$ were excluded, and the remaining data $(n=488)$ were used for this study. Fig. 1 summarizes the number of surfaces with each material per hospital.

\section{Ethical consideration}

Since the study contained no human subjects or samples, the Faculty of Health Sciences at Hokkaido University or each hospital waived the need for ethical approval, including written consent [19]. Meanwhile, the study design was explained and consents for obtaining ATP or stamp samples into hospital environments were orally obtained from all medical staffs and hospital inpatients, which intend to participate in this study. Also, privacy and confidentiality of personal information was also protected, according to the Helsinki declaration [23].

\section{Scanning electron microscopy (SEM)}

According to a previous method [24], floor material samples (approximately $1 \times 1 \mathrm{~cm}$ ) were washed in saline, fixed with $2.5 \%(\mathrm{v} / \mathrm{v})$ glutaraldehyde in phosphate-buffered saline ( $\mathrm{pH} 7.4)$ for $2 \mathrm{~h}$ at room temperature, and subsequently soaked in $2 \%(\mathrm{w} / \mathrm{v})$ osmium tetroxide for $1 \mathrm{~h}$ at $4{ }^{\circ} \mathrm{C}$. The samples were then dehydrated in ethanol, freeze-dried, and coated with osmium using a plasma osmium coater. Samples were analyzed using a Hitachi S-4800 SEM (Hitachi, Tokyo, Japan). The materials [disutilized floor samples actually used for each of the hospitals $(\mathrm{A}-\mathrm{C})]$ consisting of vinyl chloride (Hospital "B" floor material also contained antibacterial substance) were kindly provided by TOLI Corporation (Osaka, Japan) and TAJIMA ROOFINF (Tokyo, Japan). 


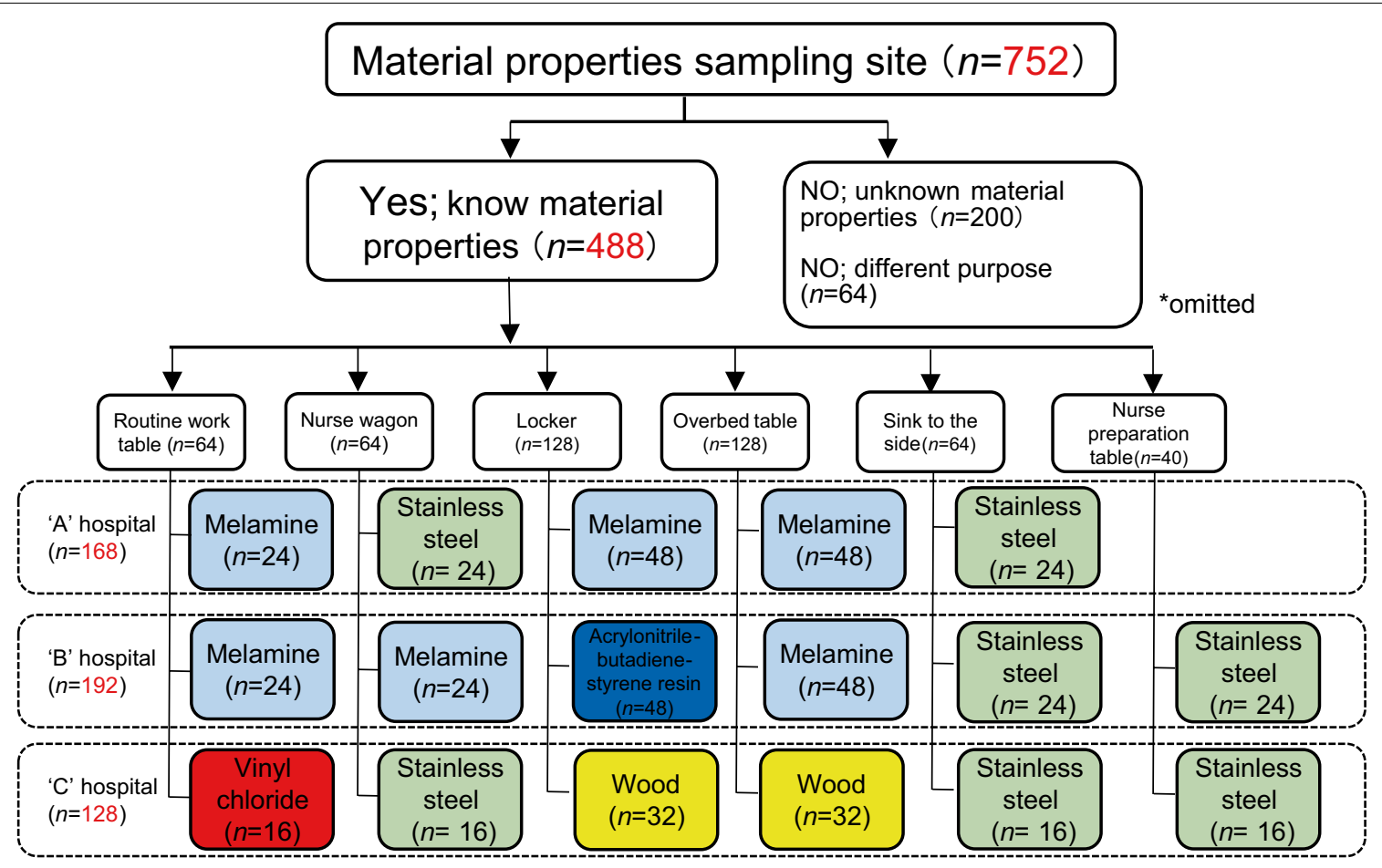

Fig. 1 Classification of material surface properties. The material surface properties of the sampling sites were divided into six categories: melamine coated, vinyl chloride, stainless steel, wood, acrylonitrile-butadiene styrene resin coated, and antibacterial vinyl chloride. *because of one missing sample, there are 751 sites with ATP data

\section{Statistical analysis}

Comparison of the data between individual material properties was assessed by one-way analysis of variance and multiple regression analysis. A $p$ value of $<0.05$ was considered statistically significant.

\section{Results}

ATP bioluminescence values, but not stamp values, differ significantly depending on material surface properties

The ATP values from high-touch places significantly differed in a material property-dependent manner (Fig. 2, $p<0.001$ ). Each of the distinct material surfaces showed the following trends. Stainless steel showed the lowest ATP values among the categories, determining the surface as low-touch place, and acrylonitrile-butadiene styrene resin coated and vinyl chloride remained neutral, indicating medium-touch places. In contrast to these, both melamine coated surface and wood surface were relatively higher than the others, although wood surface were considerably in variable.

Meanwhile, the stamp values did not significantly differ by surface, although in contrast to ATP values stamp results regardless of material properties were considerably in variable (Fig. 3, $p=0.194$ ).

Taken together, the findings indicated that in contrast to stamp values, ATP-accumulation more depends on the physical properties of the material surface such as electronic charges or roughness.

\section{Ultra-structures of vinyl chloride material surfaces (disutilized floor samples) shows considerable roughness} Vinyl chloride surfaces as well as melamine coated consisting of chemical polymers are believed to be very convenient materials, protecting from water leakage or pollution from dirty materials similar to stainless steel material. However, the data were contradictory to this notion, with moderate values of ATP test. To explore this contradiction, ultra-structures of vinyl chloride surface materials (disutilized floor samples actually used for each of the hospitals) were visualized by SEM. As a result, all the SEM images similarly revealed that there is extreme roughness on the vinyl chloride surfaces, which may allow space for colonizing microbes without noticing it (Fig. 4, high magnification). As expected, our data showed actual floor swab samples with moderate values of ATP test, indicating a dirty place (data not shown).

Thus, these data support that in contrast to stamp values, ATP-accumulation results more depend on the material surface physical properties such as roughness, suggesting that the ATP method may overestimate hospital cleanliness. 


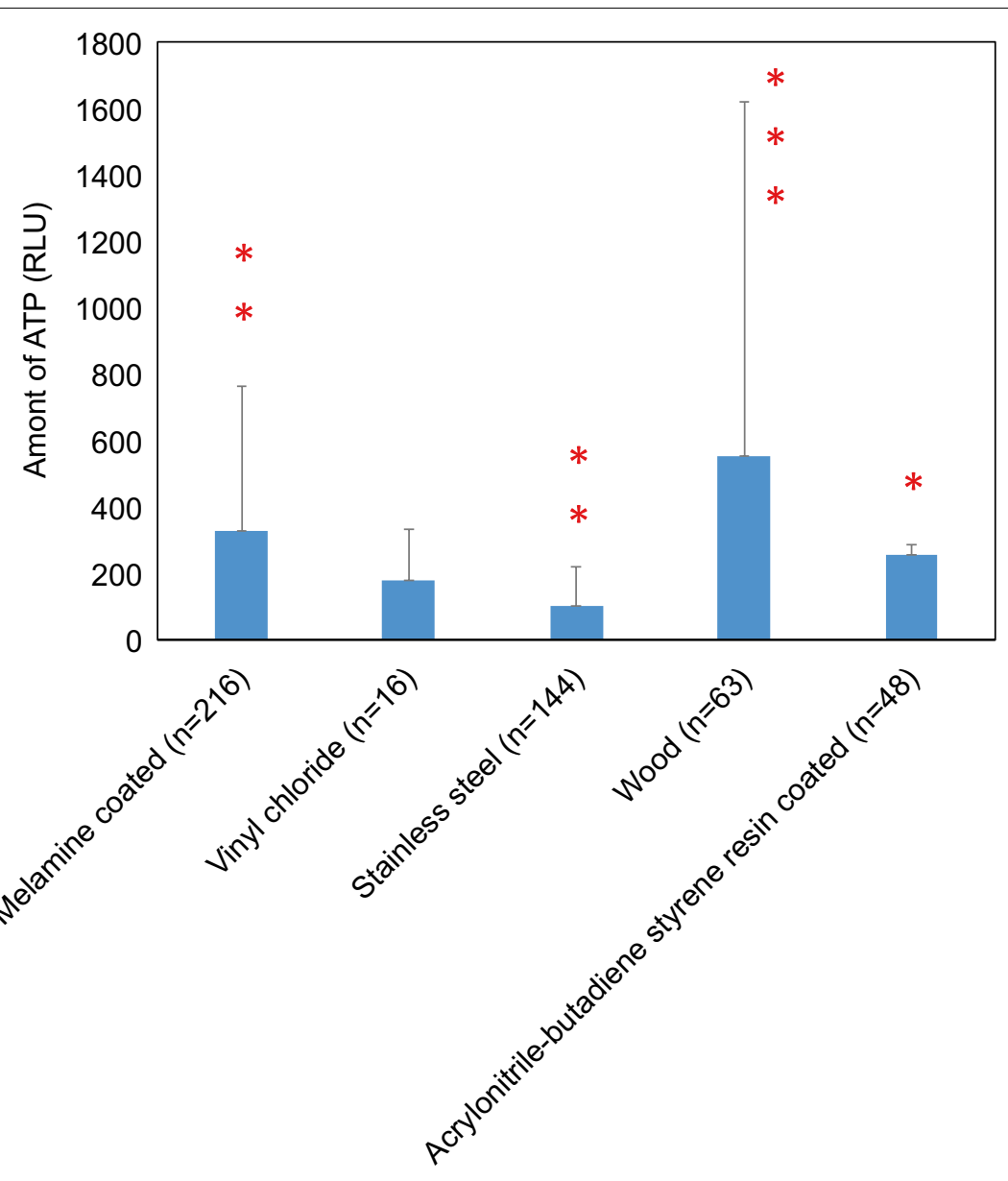

\begin{tabular}{|lrrrrr|}
\hline & \multicolumn{5}{c|}{ p valuas vs. } \\
\cline { 2 - 7 } & $\mathrm{M}$ & $\mathrm{V}$ & $\mathrm{S}$ & $\mathrm{W}$ & $\mathrm{A}$ \\
\hline Melamine coated (M) & & 0.768 & 0.000 & 0.013 & 0.893 \\
Vinyl chloride (V) & 0.768 & & 0.978 & 0.053 & 0.982 \\
Stainless steel (S) & 0.000 & 0.978 & & 0.000 & 0.335 \\
Wood (W) & 0.013 & 0.053 & 0.000 & & 0.015 \\
Acrylonitrile-butadiene styrene resin coated (A) & 0.893 & 0.982 & 0.335 & 0.015 & \\
\hline
\end{tabular}

Fig. 2 ATP amount estimated by the ATP method by each material. Values show the amount of ATP (RUL) + standard deviation/100 $\mathrm{cm}^{2} .{ }^{*} p<0.001$ vs. the other class. Asterisk numbers also indicate the number of classes with a statistically significant difference. The values in the table show $p$ values with statistical significant (Red)

\section{Discussion}

Stainless steel surfaces are believed to maintain cleanliness relatively well, with minimal attachment of organic constituents. However, our analysis revealed that even though stainless steel may be determined as clean by the ATP method, stamp values were not identical to this trend, indicating that microbial contamination does occur. Although the reason for this inconsistency is unknown, several studies have indicated that attachment of microbes on stainless steel surfaces is significantly influenced by temperature or humidity, and that stainless steel can support microbial survival [25]. Additionally, if given weeks to grow, microbes appear to have an inherent ability to colonize any stainless steel or polymeric material generally used for high-touch surfaces in hospitals [26]. Taken together with our data, we caution that ATP testing on stainless steel of hospital likely overestimates hospital cleanliness.

In contrast to stainless surface, ATP results on wood surfaces were relatively higher than those of the other 


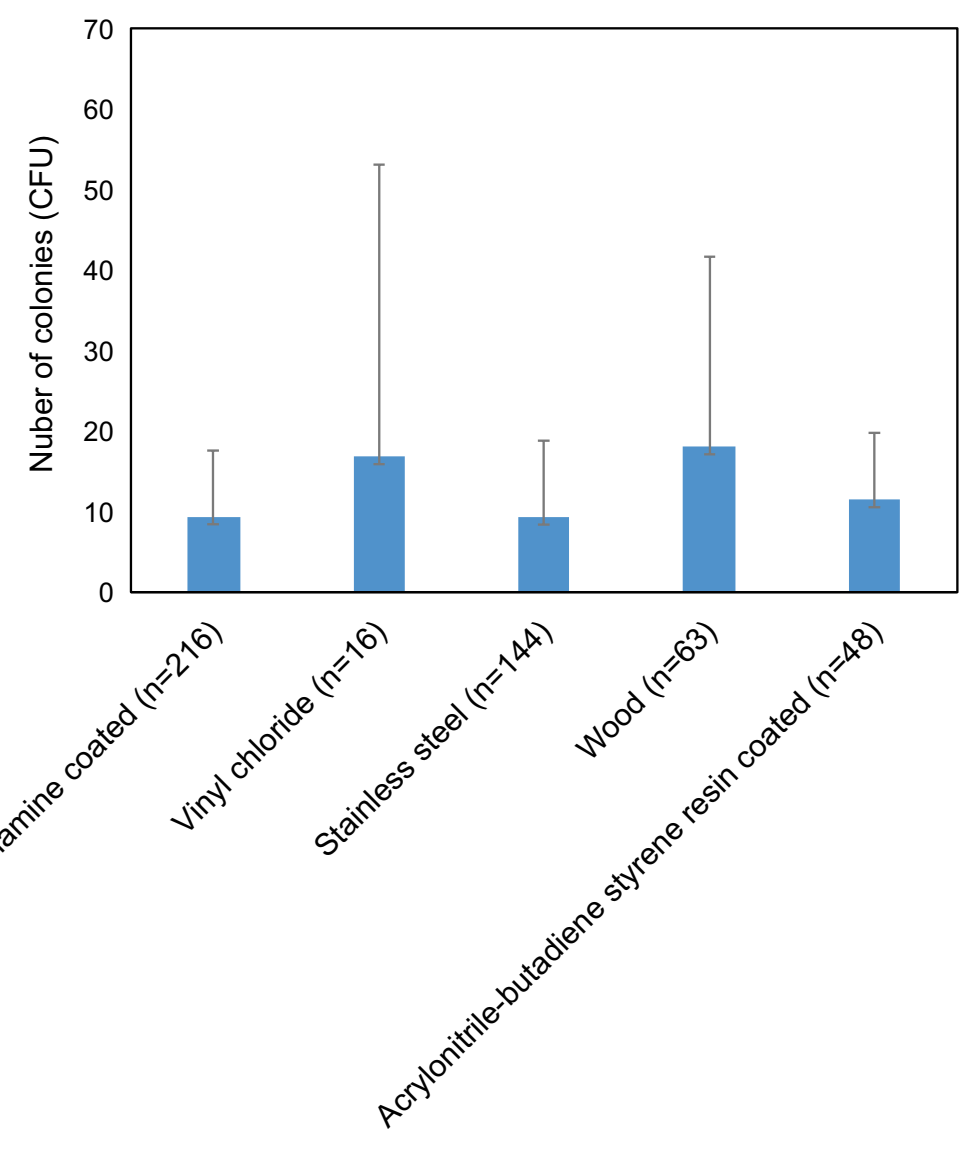

\begin{tabular}{|lrrrrr|}
\hline & \multicolumn{5}{c}{ p valuas vs. } \\
\cline { 2 - 7 } & $\mathrm{M}$ & $\mathrm{V}$ & $\mathrm{S}$ & $\mathrm{W}$ & $\mathrm{A}$ \\
\hline Melamine coated (M) & & 0.812 & 0.761 & 0.152 & 0.749 \\
Vinyl chloride (V) & 0.812 & & 0.977 & 1.000 & 0.998 \\
Stainless steel (S) & 0.761 & 0.977 & & 0.682 & 0.995 \\
Wood (W) & 0.152 & 1.000 & 0.682 & & 0.957 \\
Acrylonitrile-butadiene styrene resin coated (A) & 0.749 & 0.998 & 0.995 & 0.957 & \\
\hline
\end{tabular}

Fig. 3 Comparison of CFU amount estimated by the stamp method by each material. Values show amount of CFU + standard deviation $/ 10 \mathrm{~cm}^{2}$. The values in the table show $p$ values

materials even though considerably in variable, indicating that wood surfaces were more high-touched than vinyl chloride or stainless steel. However, the exact reason why ATP values on wood surfaces were considerably in variable remains unknown. Meanwhile, we found the fact that all wood material surfaces assessed into the study were coated with melamine polymer, although the coating amount or timing could not be found out. Therefore, it cannot deny that difference of the polymer coating degree to protect wood surface could influence the ATP evaluation. Thus, we also caution that ATP values on wood surface are likely to be inaccurate, and less wood material in hospitals may be useful to improve hospital cleanliness.

In general, materials consisting of vinyl chloride as well as melamine are chemical polymers and are commonly used in daily goods such as tables, bed frames, dishes, and floors. Such chemical polymers are believed to be very convenient materials, protecting from water leakage or pollution from dirty materials, which presumably makes their surfaces easier to keep free of microbial contamination. However, the data were contradictory to 


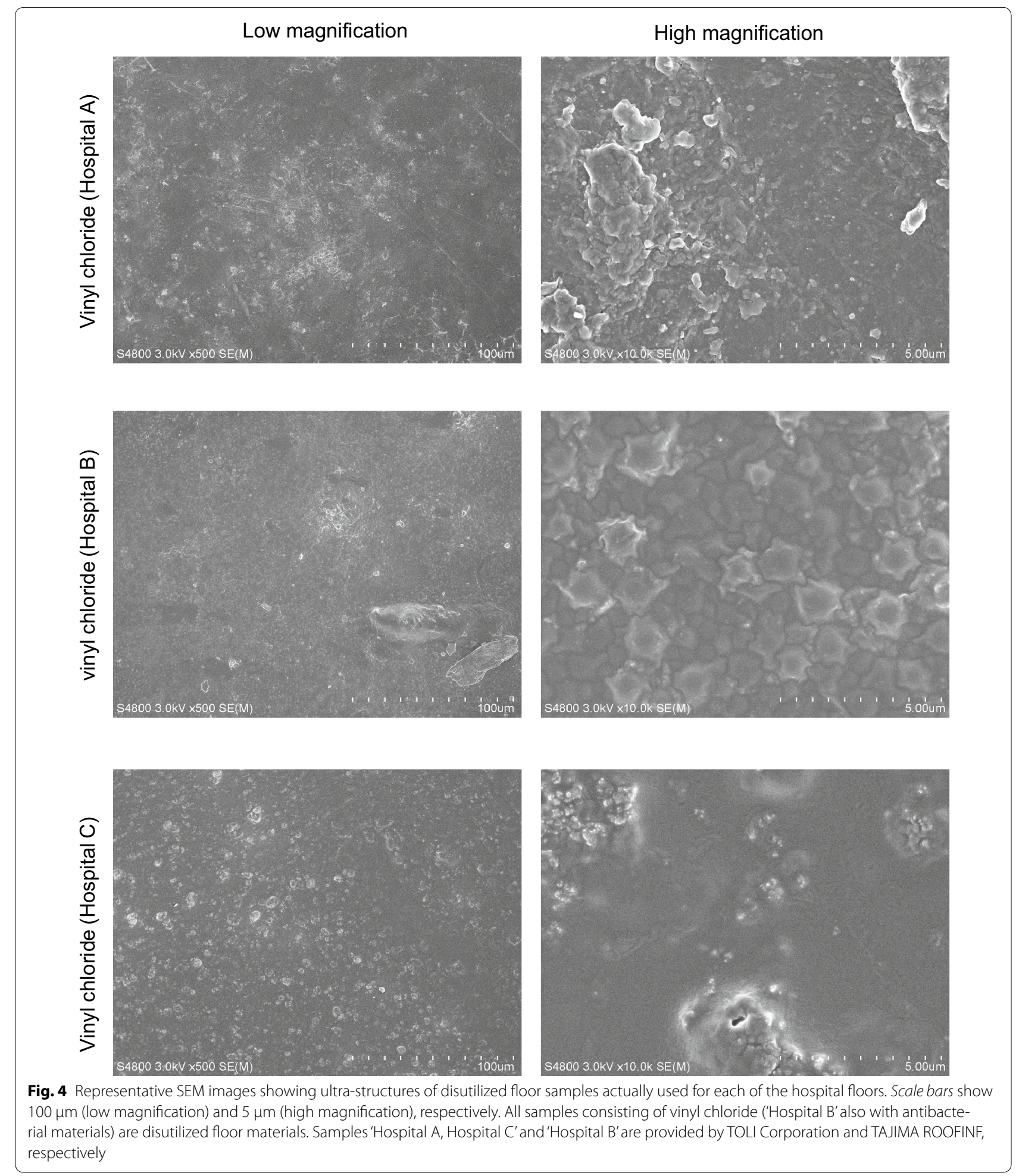

this notion, with moderate values of ATP test, implying the presence of unknown physical factor such as roughness with changes depending on wears, which provides microbes with hiding places. We therefore attempted to see a roughness degree of the material surface by the observation with SEM. As expected, the SEM images revealed an extreme roughness on the vinyl chloride surfaces, allowing space for colonizing microbes, easily 
altered by a degree of wears. In addition, it cannot deny that since ATP assessment is done by hard swab included in the commercial kit, sampling on rough surfaces may inefficiently collect organic material. Thus, although the data are limited, the observation provides us with a caution that cleanliness of surfaces containing chemical polymers with a distinct degree of roughness may be overestimated, and suggests a need for more adequate hospital cleanliness monitoring protocols.

The reason why ATP values were significantly different in a material property-dependent manner is currently unknown. Meanwhile, environmental ATP is sufficiently stable to ensure environmental cleaning in health care, and has been experimentally demonstrated that in contrast to liquid suspension, ATP on dried surfaces remained very stable for $29 \mathrm{~h}$ [27]. Taken together with this, our results suggest that while in daily monitoring assessments ATP deterioration is minimal [19], ATP accumulation may dramatically change in a material property-dependent manner. Thus, use of the ATP test alone may lead to an overestimation of hospital cleanliness when used for immediate feedback of cleaning coverage.

Benchmark values of ATP and stamp evaluation into assessing environmental cleanliness have been already proposed as $250 \mathrm{RLU} / 10 \mathrm{~cm}^{2}$ and $2.5-5 \mathrm{CFU} / \mathrm{cm}^{2}$, respectively [12, 28]. Meanwhile, as described previously [19], our system revealed that the detection limits of the ATP method (according to the spiked experiment with Escherichia coli, Staphylococcus aureus, and Bacillus subtilis) and stamp method [according to taking samples from student tables $(n=19)$ in a lecture room of our department during vacation] were estimated at 12.7 $\mathrm{RLU} / 10 \mathrm{~cm}^{2}$ and $0.5 \mathrm{CFU} / \mathrm{cm}^{2}$, respectively. Thus, although our detection limits were estimated with accuracy, these appeared to be relatively low when compared with the previous benchmark values. Furthermore, although the exact reason why its contradiction occurred also remains unknown at this time, the recent observation assessing hospital cleanliness in Japan with an estimated benchmark values similar to our detection limits, supported our data to be accurate [29].

According to cleaning and hand hygiene protocols, hospital environmental surfaces are believed to maintain minimal microbial contamination, and clean surfaces contribute to reducing infectious diseases in hospitals. However, hospital-acquired infections continue to result in a significant loss of life and high cost (e.g., the United States healthcare system estimated $\$ 45$ billion annually) [30]. In support of this, our previous data have revealed that ongoing daily hospital cleanliness is not sufficient in hospitals with a tendency toward long-term care in Japan [19]. Thus, hospital cleanliness must be reevaluated from the point of view of material surfaces for preventing hospital-acquired infections, to establish more accurate cleanliness protocols and accurate monitoring systems.

\section{Conclusion}

Taken together, we conclude that material surface properties must be considered in evaluating high-touch places with ATP values, and provide a strong warning to the possibility of overestimating hospital cleanliness with the use of the ATP test alone.

\section{Abbreviations}

ATP: adenosine triphosphate; SEM: scanning electron microscope; CFU: colony-forming units.

\section{Authors' contributions}

TS and RY performed the data analysis and sampling. TS, RY and JM previously obtained the sampling data (both ATP and stamp). SN and MY performed technical assistance in SEM imaging. TS, RY and HY conducted the statistical analysis. TS, RY, and HY designed the study. SY and HY supervised the practical work and data management. HY wrote the manuscript. All authors read and approved the final manuscript.

\section{Author details}

${ }^{1}$ Department of Fundamental Nursing, Faculty of Health Sciences, Hokkaido University, Sapporo, Japan. ${ }^{2}$ Laboratory of Morphology and Image Analysis, Biomedical Research Center, Juntendo University Graduate School of Medicine, Tokyo, Japan. ${ }^{3}$ Department of Medical Laboratory Science, Faculty of Health Sciences, Hokkaido University, Nishi-5 Kita-12 Jo, Kita-ku, Sapporo, Hokkaido 060-0812, Japan.

\section{Acknowledgements}

We thank the staff members of the Departments of Medical Laboratory Science and Nursing, Faculty of Health Sciences, Hokkaido University, for their assistance throughout this study.

\section{Competing interests}

The authors declare that they have no competing interests.

\section{Funding}

This study was supported by grants-in-aid for Japan Society for the Promotion of Science (JSPS) fellows and scientific research from KAKENHI, grant numbers $25670902,21590474,24659194$, and 24117501. The funders had no role in study design, data collection and analysis, decision to publish, or preparation of the manuscript.

Received: 1 September 2015 Accepted: 26 November 2015 Published online: 21 December 2015

\section{References}

1. Boyce JM. Environmental contamination makes an important contribution to hospital infection. J Hosp Infect. 2007:65(Suppl. 2):50-4.

2. Dettenkofer M, Spencer RC. Importance of environmental contamination-a critical view. J Hosp Infect. 2007;65(Suppl. 2):55-7.

3. Fraise AP. Decontamination of the environment. J Hosp Infect. 2007;65(Suppl. 2):58-9.

4. Lemmes SW, Hafner H, Zolldann D, Stanzel S, Lutticken R. Distribution of multi-resistant gram-negative versus gram-positive bacteria in the hospital inanimate environment. J Hosp Infect. 2004;56:191-7.

5. Hayden MK, Bonten MJM, Blom DW, Lyle EA, van de Vijver DAMC, Weinstein RA. Reduction in acquisition of vancomycin-resistant enterococcus after enforcement of routine environmental cleaning measures. Clin Infect Dis. 2006:42:1552-60. 
6. Hardy KJ, Oppenheim BA, Gossain S, Gao F, Hawkey PM. A study of the relationship between environmental contamination with methicillinresistant Staphylococcus aureus (MRSA) and patients' acquisition of MRSA. Infect Cont Hosp Epidemiol. 2006;27:127-32.

7. Dancer SJ, White LF, Lamb J, Girvan EK, Robertson C. Measuring the effect of enhanced cleaning in a UK hospital: a prospective cross-over study. BMC Med. 2009;7:28.

8. Liz. Essential practice for infection prevention and control, Guidance for nursing staff. Royal College of Nursing. 2012.

9. NHMRC. Australian guidelines for the prevention and control of infection in healthcare. Commonwealth of Australia. 2010.

10. Rutala WA. Guideline for disinfection and sterilization in healthcare facilities, 2008. Centers for Disease Control and Prevention (US). 2008.

11. Moore G, Smyth D, Singleton J, Wilson P. The use of adenosine triphosphate bioluminescence to assess the efficacy of a modified cleaning program implemented within an intensive care setting. Am J Infect Control. 2010;38:617-22

12. Boyce JM, Havill NL, Dumigan DG, Golebiewski M, Balogun O, Rizvani R. Monitoring the effectiveness of hospital cleaning practices by use of an adenosine triphosphate bioluminescence assay. Infect Control Hosp Epidemiol. 2009;30:678-84.

13. Andersen BM, Rasch M, Kvist J, Tollefsen T, Lukkassen R, Sandvik L, Welo A. Floor cleaning: effect on bacteria and organic materials in hospital rooms. J Hosp Infect. 2009;71:57-65.

14. Malik RE, Cooper RA, Griffith CJ. Use of audit tools to evaluate the efficacy of cleaning systems in hospitals. Am J Infect Control. 2003;31:181-7.

15. Griffith CJ, Cooper RA, Gilmore J, Davies C, Lewis M. An evaluation of hospital cleaning regimes and standards. J Hosp Infect. 2000;45:19-28.

16. Dolan A, Bartlett M, McEntee B, Creamer E, Humphreys H. Evaluation of different methods to recover meticillin-resistant Staphylococcus aureus from hospital environmental surfaces. J Hosp Infect. 2011;79:227-30.

17. Cheng KL, Boost MV, Chung JW. Study on the effectiveness of disinfection with wipes against methicillin-resistant Staphylococcus aureus and implications for hospital hygiene. Am J Infect Control. 2011;39:577-80.

18. Nandalal P, Somashekar RK. Prevalence of Staphylococcus aureus and Pseudomonas aeruginosa in indoor air flora of a district hospital, Mandya, Karnataka. J Environ Biol. 2007;28:197-200.

19. Watanabe R, Shimoda T, Yano R, Hayashi Y, Nakamura S, Matsuo J, Yamaguchi H. Visualization of hospital cleanliness in three Japanese hospitals with a tendency toward long-term care. BMC Res Notes. 2014;7:121.
20. Wang Z, Shen Y, Haapasalo M. Dental materials with antibiofilm properties. Dent Mater. 2014;30:e1-16.

21. Taylor J, Lai KM, Davies M, Clifton D, Ridley I, Biddulph P. Flood management: prediction of microbial contamination in large-scale floods in urban environments. Environ Int. 2011;37:1019-29.

22. Veerachamy S, Yarlagadda T, Manivasagam G, Yarlagadda PK. Bacterial adherence and biofilm formation on medical implants: a review. Proc Inst Mech Eng H. 2014;228:1083-99.

23. Moore G, Smyth D, Singleton J, Wilson P. The use of adenosine triphosphate bioluminescence to assess the efficacy of a modified cleaning program implemented within an intensive care setting. Am J Infect Control. 2010;38:617-22.

24. Okude M, Matsuo J, Nakamura S, Kawaguchi K, Hayashi Y, Sakai H, Yoshida M, Takahashi K, Yamaguchi H. Environmental chlamydiae alter the growth speed and motility of host acanthamoebae. Microbes Environ. 2012;27:423-9.

25. Verran J, Whitehead K. Factors affecting microbial adhesion to stainless steel and other materials used in medical devices. Int J Artif Organs. 2005;28:1138-45.

26. Michels HT, Wilks SA, Noyce JO, Keevil CW. Copper alloys for humaninfectious disease control. In: Proceedings of the Materials Science and Technology Conference, Copper for the 21st Century Symposium. Pittsburgh: 25 to 28 September, 2005. p. 1-11.

27. Alfa MJ, Olson N, Murray BL. Adenosine tri-phosphate (ATP)-based cleaning monitoring in health care: how rapidly does environmental ATP deteriorate? J Hosp Infect. 2015. doi:10.1016/j.jhin.2015.01.020.

28. Dancer SJ. How do we assess hospital cleaning? A proposal for microbiological standards for surface hygiene in hospitals. J Hosp Infect. 2004;56:10-5.

29. Kajigaya N, Hirose Y, Koike S, Fujita T, Yokota N, Hata S, Ikenaga M, Kobayashi N, Takahashi T. Assessment of contamination using an ATP bioluminescence assay on doorknobs in a university-affiliated hospital in Japan. BMC Res Notes. 2015:8:352.

30. Sharpe PA, Schmidt MG. Control and mitigation of healthcare-acquired infections: designing clinical trials to evaluate new materials and technologies. HERD. 2011;5:94-115.

\section{Submit your next manuscript to BioMed Central and we will help you at every step:}

- We accept pre-submission inquiries

- Our selector tool helps you to find the most relevant journal

- We provide round the clock customer support

- Convenient online submission

- Thorough peer review

- Inclusion in PubMed and all major indexing services

- Maximum visibility for your research

Submit your manuscript at www.biomedcentral.com/submit
() Biomed Central 